\title{
PIERO ontology for analysis of biochemical transformations: Effective implementation of reaction information in the IUBMB enzyme list
}

\author{
Masaaki Kotera*,

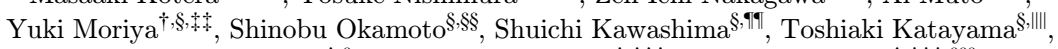 \\ Toshiaki Tokimatsu ${ }^{\dagger, \S, * * *}$, Minoru Kanehisa ${ }^{\dagger, \dagger \dagger}$ and Susumu Goto ${ }^{\dagger,+1+, \S \S \S}$ \\ * Graduate School of Bioscience and Biotechnology \\ Tokyo Institute of Technology \\ 2-12-1 Ookayama, Meguro-ku, Tokyo 152-8550, Japan \\ ${ }^{\dagger}$ Bioinformatics Center, Institute for Chemical Research \\ Kyoto University, Gokasho, Uji, Kyoto 611-0011, Japan \\ Graduate School of Biological Sciences \\ Nara Institute of Science and Technology (NAIST) \\ 8916-5 Takayama, Ikoma, Nara 630-0192, Japan \\ $\S$ Database Center for Life Science \\ Research Organization of Information and Systems \\ 178-4-4 Wakashiba, Kashiwa, Chiba 277-0871, Japan \\ Tmaskot@bio.titech.ac.jp

Received 14 July 2014

Revised 12 September 2014

Accepted 12 September 2014

Published 10 November 2014

Genomics is faced with the issue of many partially annotated putative enzyme-encoding genes for which activities have not yet been verified, while metabolomics is faced with the issue of many putative enzyme reactions for which full equations have not been verified. Knowledge of enzymes has been collected by IUBMB, and has been made public as the Enzyme List. To date, however, the terminology of the Enzyme List has not been assessed comprehensively by bioinformatics studies. Instead, most of the bioinformatics studies simply use the identifiers of the

$\S \S$ Corresponding author.

१ๆ First and second author contributed equally to the paper. 


\begin{abstract}
enzymes, i.e. the Enzyme Commission (EC) numbers. We investigated the actual usage of terminology throughout the Enzyme List, and demonstrated that the partial characteristics of reactions cannot be retrieved by simply using EC numbers. Thus, we developed a novel ontology, named PIERO, for annotating biochemical transformations as follows. First, the terminology describing enzymatic reactions was retrieved from the Enzyme List, and was grouped into those related to overall reactions and biochemical transformations. Consequently, these terms were mapped onto the actual transformations taken from enzymatic reaction equations. This ontology was linked to Gene Ontology (GO) and EC numbers, allowing the extraction of common partial reaction characteristics from given sets of orthologous genes and the elucidation of possible enzymes from the given transformations. Further future development of the PIERO ontology should enhance the Enzyme List to promote the integration of genomics and metabolomics.
\end{abstract}

Keywords: Enzyme; reaction; transformation; ontology.

\title{
1. Introduction
}

Recent development of high-throughput techniques has promoted large-scale analysis of molecular biological data, including genomic and metabolomic data. However, much of these data have been computationally predicted, but have not been verified experimentally. ${ }^{1}$

First, genomics studies have identified a vast number of putative genes, but many of these genes have only been partially annotated. For many putative enzymeencoding genes, annotations are given using Enzyme Commission (EC) numbers ${ }^{2}$ or Gene Ontology (GO) identifiers. ${ }^{3}$ Molecular functions in GO correspond completely to EC numbers; thus these identifiers are not substantially different. However, EC numbers are not applicable for annotating the genes (or gene products) that mediate unknown reactions, because they are assigned only to those enzymes for which overall reaction equations have been experimentally verified.

Second, metabolomics studies have implicated many biochemical compounds (metabolites) but how they are synthesized remains unknown. ${ }^{4}$ Putative pathways have been considered for such compounds in many studies, where the putative intermediate compounds are connected to each other by partial (incomplete) reaction equations. However, EC numbers are not applicable for the partial reactions either, for the same reason as described above.

It is important to clarify what is meant by "partial reaction" in this paper. A reaction may be divided into multiple reaction steps, where the product of one step becomes the substrate of the next step. Such reaction steps are not the focus of this paper. The partial reaction described in this paper is the substrate-product relationship, also referred to as the "transformation", in which the role of the other molecules is unclear (Fig. 1(a)).

Here, we propose that organizing the terminology of enzyme reactions in the framework of computer-aided ontology ${ }^{5}$ would help not only to characterize and identify the enzymes that possibly mediate partial reactions of interest, but also to systematically extract the common biochemical characteristics from a group of enzyme-encoding genes. 
(a) a transformation (partial reaction)<smiles>CC(=O)NCCCC[C@H](N)C(=O)O</smiles>

(b) reactions (overall reactions)
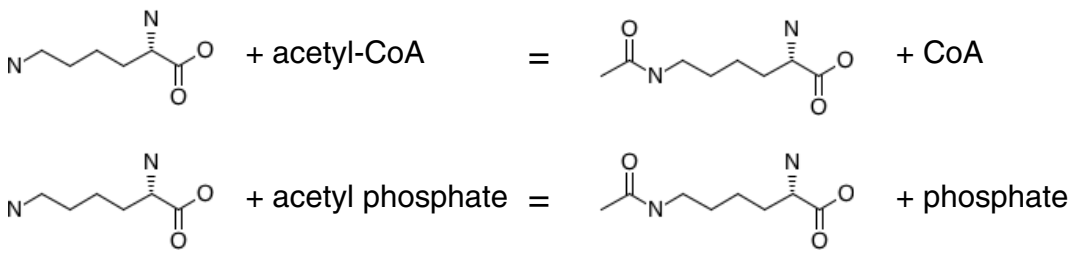<smiles>[13CH3]OC(=O)[C@@H](N)CCCCN</smiles><smiles>C=C[C@@H](N)C(=O)OC(C)=O</smiles>

Fig. 1. A transformation and the corresponding reactions. The use of "a transformation" only concerns a change in the substrate, whereas the use of "a reaction" concerns all of the substrates and all of the products. (a) In organic chemistry, this transformation describes the change in the substrate, irrespective of the acetylating reagent. In metabolomics, this transformation describes the substrate-product relationship, or the partial reaction, for which the acetylating reagent is not known yet. (b) These reactions share the same transformation. The difference lies in the acetylating reagents.

\subsection{Related works}

The International Union of Pure and Applied Chemistry (IUPAC) and the International Union of Biochemistry and Molecular Biology (IUBMB) provide systematic nomenclature for naming chemical reactions ${ }^{6}$ and enzymes, ${ }^{2}$ respectively. The IUBMB's Enzyme List is the most valuable resource of experimentally verified enzymes, and it provides EC numbers, four-digit identifiers (IDs), to distinguish enzymes. IUBMB classifies enzymes into six EC classes, i.e. EC1 oxidoreductases, EC2 transferases, EC3 hydrolases, EC4 lyases, EC5 isomerases, and EC6 ligases, which are indicated by the first digit of the EC numbers. The purpose of establishing the Enzyme List was the development of a standard vocabulary for naming enzymes. Originally, EC numbers were given only to the enzymes for which reactions had been characterized experimentally. With the increase in the number of genome projects, EC numbers have also been used for the annotation of putative genes on the basis of homology information, and not only on experimental characterization.

BioPortal $^{5}$ contains several ontologies that deal with enzymatic functions, but only via EC numbers. GO, on the other hand, is the most widely used dictionary for terminology related to gene functions. ${ }^{3}$ GO classifies gene functions, among which enzymatic functions are described using EC numbers. ChEBI and REX ontologies ${ }^{7}$ also deal with processes that involve chemistry, but they are based on GO (i.e. based on EC numbers). Enzyme Mechanism Ontology (EMO) describes enzyme reactions 
and their reaction steps, but does not deal with the partial reactions that we describe in this study. Name Reaction Ontology (RXNO) considers organic name reactions but does not deal with enzyme reactions.

\subsection{Scope of PIERO}

Suppose a situation in which two compounds are possibly converted to each other via enzymatic catalysis (Fig. 1(a)), but for which the enzyme has not yet been identified. In fact, three enzymes may be involved in catalyzing this biochemical transformation (Fig. 1(b)). However, in the supposed situation, it is not known which of the three is involved, and it is also possible that none of the three is actually involved (an unknown reaction may occur).

It is important to note that EC numbers are not given to partial reactions (or transformations), and also that partial EC numbers do not necessarily describe the full characteristics of such transformations. For example, the partial reaction in Fig. 1(a) can be described in a sentence such as "This reaction involves an acetylation of an amine group, yielding an $\mathrm{N}$-acetyl group". Although the three reactions in Fig. 1(b) are actually known, if we assume that they are unknown, they are assigned partial EC numbers EC2.3.1 or EC3.5.1. However, an annotation such as "This reaction is possibly catalyzed by the enzymes belonging to EC2.3.1 or EC3.5.1" causes loss of much information, because EC2.3.1 or EC3.5.1 do not provide any specific terminology such as acetylation, amine group, or $N$-acetyl group. The EC numbers only provide broader meanings; EC2.3.1 is defined as "transferases that transfer acyl groups other than aminoacyl groups", and EC3.5.1 is defined as "hydrolases acting on carbon-nitrogen bonds, other than peptide bonds, in linear amides". It is therefore challenging to describe the characteristics of enzymes with such incomplete equations.

Suppose another situation in which three enzymes mediate three reactions (Fig. 1(b)), from which we want to obtain some common characteristics. It is clear that the three reactions have a substrate-product relationship in common (Fig. 1(a)), however, this would be much more difficult to find if the number of enzymes (or reactions) was larger. The possible answer using EC numbers would be "Two of them belong to EC2.3.1, and one of them belongs to EC3.5.1", which may suggest erroneously that there are no common characteristics among the three reactions.

The reason for the failure to extract these characteristics, which may occur frequently in bioinformatics studies, is that we only use EC numbers. EC numbers are merely the identifiers of enzymes, and greater detail is provided in the Enzyme List in natural language.

We thus propose an ontology, named PIERO (Enzyme Reaction Ontology for annotating Partial Information of biochemical transformation), which deals with the terminology corresponding to overall and partial biochemical changes in enzymatic reactions, using biochemical terms (such as acetylation, hydrolysis, amine group, 
amide bond), the names of compounds (such as those in the substrate-product relationship), and the relationships among such terms. The concept of this study follows the IUPAC recommendations of $1988,{ }^{6}$ which distinguish a "transformation" (substrate-product relationship) from a "reaction" (overall reaction) clearly for the purpose of naming organic chemical transformations. In this study, we used this concept, incorporated the terminology in the IUBMB's Enzyme List (which is slightly different from that of IUPAC), and also separated the terminology into that representing reactions and transformations. This ontology was mapped onto KEGG reactions and the related entries, accompanied by linking with GO and EC numbers, thereby allowing the extraction of the common partial characteristics from the given sets of orthologous genes and the elucidation of possible enzymes from the given partial reactions.

\section{Materials and Methods}

Enzyme reactions, chemical structures of compounds and other enzyme-related data were retrieved from the KEGG database. ${ }^{8}$

The substructure-product relationship was taken from KEGG RPAIR database, ${ }^{9}$ where substrate-product relationships describe the transformations in the reactions. Taking a reaction $G l c+A T P=G l c-6-P+A M P$, for example, the "main" types of transformations are "Glc $->$ Glc- 6 - $P$ " (conserving glucose residue) and "ATP $>>$ $A M P$ " (conserving AMP residule). The pair "ATP->Glc-6-P" is defined as a "trans" type of transformation, representing the transferred phosphate group. The pair "Glc $->A M P$ " is not defined as a transformation because it does not conserve any atoms.

The relationships between the transformations and the corresponding transformation patterns were taken from the KEGG RCLASS database. ${ }^{10}$

The relationships between biochemical substructures and the corresponding names were retrieved from the BiSSCat database ${ }^{11}$ using KEGG Chemical Functionand-Substructures (KCF-S). ${ }^{12}$

These data were converted to the Resource Description Framework (RDF), where the relationships were described by the triad of subjects, predicates, and objects. ${ }^{13}$

\section{Results and Discussion}

\subsection{Vocabulary appearance in the Enzyme List}

Generally, an enzyme has a systematic name and more than one accepted names, and enzymes are named according to the reactions they catalyze. The Enzyme List terminology of the reactions and the transformations is represented with the suffix "-ase" when the terminology represents the main characteristics of the reaction ( e.g. decarboxylase). When the terminology does not represent the main characteristics, but when it is important to distinguish its action from that of other enzymes, the suffix "-ing" is used (e.g. decarboxylating). 
We counted the appearance of EC numbers that contain the terms including the suffixes -ase and -ing (Tables 1 and 2, respectively). Some terms (such as phosphohydrolase, amidohydrolase, proteinase, and dioxygenase) only appear in one EC class (indicated by the first digit of EC numbers). However, many terms (such as cyclase

Table 1. Top 40 terms with the suffix "-ase" in the Enzyme List.

\begin{tabular}{|c|c|c|c|c|c|c|c|}
\hline & \multirow[b]{2}{*}{ Total } & \multicolumn{6}{|c|}{ The first digit of the EC numbers } \\
\hline & & EC1 & $\mathrm{EC} 2$ & EC3 & EC4 & EC5 & EC6 \\
\hline Oxidoreductase & 1480 & 1474 & 0 & 0 & 4 & 2 & 0 \\
\hline Synthase & 748 & 113 & 295 & 16 & 202 & 67 & 55 \\
\hline Lyase & 618 & 9 & 37 & 4 & 545 & 21 & 2 \\
\hline Dehydrogenase & 580 & 572 & 5 & 3 & 0 & 0 & 0 \\
\hline Reductase & 399 & 389 & 6 & 3 & 1 & 0 & 0 \\
\hline Hydrolase & 283 & 1 & 3 & 264 & 14 & 1 & 0 \\
\hline Methyltransferase & 269 & 1 & 268 & 0 & 0 & 0 & 0 \\
\hline Synthetase & 263 & 12 & 97 & 3 & 22 & 10 & 119 \\
\hline Phosphotransferase & 260 & 0 & 257 & 1 & 0 & 2 & 0 \\
\hline Transferase & 216 & 5 & 203 & 6 & 2 & 0 & 0 \\
\hline Kinase & 206 & 0 & 203 & 3 & 0 & 0 & 0 \\
\hline Oxidase & 196 & 194 & 0 & 0 & 1 & 1 & 0 \\
\hline Phosphohydrolase & 191 & 0 & 0 & 191 & 0 & 0 & 0 \\
\hline Monooxygenase & 179 & 178 & 1 & 0 & 0 & 0 & 0 \\
\hline Ligase & 176 & 0 & 6 & 3 & 3 & 0 & 164 \\
\hline Hydroxylase & 171 & 170 & 1 & 0 & 0 & 0 & 0 \\
\hline Glucosyltransferase & 128 & 0 & 124 & 1 & 0 & 3 & 0 \\
\hline Amidohydrolase & 118 & 0 & 0 & 118 & 0 & 0 & 0 \\
\hline Proteinase & 116 & 0 & 0 & 116 & 0 & 0 & 0 \\
\hline Dioxygenase & 111 & 111 & 0 & 0 & 0 & 0 & 0 \\
\hline Phosphatase & 106 & 0 & 4 & 102 & 0 & 0 & 0 \\
\hline Aminotransferase & 100 & 1 & 96 & 0 & 1 & 1 & 1 \\
\hline Acetyltransferase & 99 & 2 & 95 & 2 & 0 & 0 & 0 \\
\hline Decarboxylase & 95 & 10 & 1 & 0 & 84 & 0 & 0 \\
\hline Isomerase & 95 & 2 & 4 & 2 & 3 & 84 & 0 \\
\hline Transaminase & 89 & 0 & 87 & 0 & 2 & 0 & 0 \\
\hline Dehydratase & 87 & 0 & 0 & 1 & 86 & 0 & 0 \\
\hline Protease & 82 & 0 & 0 & 82 & 0 & 0 & 0 \\
\hline Mutase & 71 & 1 & 0 & 0 & 1 & 69 & 0 \\
\hline Peptidase & 70 & 0 & 0 & 70 & 0 & 0 & 0 \\
\hline Acyltransferase & 69 & 0 & 67 & 1 & 1 & 0 & 0 \\
\hline ATPase & 67 & 0 & 0 & 67 & 0 & 0 & 0 \\
\hline Oxygenase & 58 & 54 & 2 & 0 & 2 & 0 & 0 \\
\hline Cyclase & 55 & 2 & 5 & 1 & 31 & 14 & 2 \\
\hline Deaminase & 53 & 4 & 1 & 38 & 10 & 0 & 0 \\
\hline Endopeptidase & 52 & 0 & 0 & 52 & 0 & 0 & 0 \\
\hline Aldolase & 48 & 0 & 4 & 0 & 44 & 0 & 0 \\
\hline Epimerase & 45 & 3 & 0 & 2 & 0 & 40 & 0 \\
\hline Esterase & 45 & 0 & 0 & 45 & 0 & 0 & 0 \\
\hline Aminohydrolase & 45 & 0 & 0 & 44 & 1 & 0 & 0 \\
\hline
\end{tabular}

Note: The numbers of EC numbers that contain the respective "-ase"-form terms were counted. If an EC number had two names and both contained "decarboxylase", the term "decarboxylase" was counted once only. 
Table 2. Top 20 terms with the suffix "-ing" in the Enzyme List.

\begin{tabular}{lrrrrrrr}
\hline & & \multicolumn{5}{c}{ The first digit of the EC numbers } \\
\cline { 2 - 8 } & Total & EC1 & EC2 & EC3 & EC4 & EC5 & EC6 \\
\hline Hydroxylating & 225 & 225 & 0 & 0 & 0 & 0 & 0 \\
Cyclizing & 164 & 14 & 26 & 4 & 89 & 29 & 2 \\
Phosphorylating & 115 & 7 & 104 & 0 & 1 & 0 & 3 \\
Decarboxylating & 103 & 63 & 26 & 7 & 7 & 0 & 0 \\
Deaminating & 74 & 49 & 1 & 3 & 21 & 0 & 0 \\
Hydrolysing & 68 & 5 & 27 & 14 & 5 & 3 & 14 \\
Decyclizing & 60 & 32 & 0 & 8 & 1 & 19 & 0 \\
Transporting & 44 & 4 & 0 & 40 & 0 & 0 & 0 \\
Cleaving & 35 & 21 & 0 & 12 & 1 & 1 & 0 \\
Pyruvate-forming & 32 & 2 & 0 & 0 & 30 & 0 & 0 \\
Reducing & 25 & 9 & 7 & 7 & 2 & 0 & 0 \\
Phosphate-forming & 25 & 0 & 0 & 1 & 24 & 0 & 0 \\
Acetylating & 19 & 9 & 8 & 0 & 2 & 0 & 0 \\
Demethylating & 17 & 17 & 0 & 0 & 0 & 0 & 0 \\
Thioester-hydrolysing & 17 & 0 & 17 & 0 & 0 & 0 & 0 \\
Alpha-hydroxylating & 15 & 15 & 0 & 0 & 0 & 0 & 0 \\
Acylating & 15 & 13 & 2 & 0 & 0 & 0 & 0 \\
Isomerizing & 13 & 3 & 3 & 3 & 2 & 2 & 0 \\
Diphosphate-forming & 13 & 0 & 3 & 3 & 1 & 0 & 6 \\
Transferring & 12 & 5 & 4 & 2 & 1 & 0 & 0 \\
\hline
\end{tabular}

Note: The numbers of EC numbers that contain the respective "-ing"-form terms were counted. If an EC number had two names and both contained "decarboxylating", the term "decarboxylating" was counted once only.

and cyclizing), even those that define the six EC classes (oxidoreductase, transferase, hydrolase, lyase, isomerase, and ligase), appear in two or more EC classes.

This result implied that using partial EC numbers causes loss of information. For example, the partial EC number EC4.1.1 is defined as carboxy-lyases (synonym of decarboxylases) and it contains 84 decarboxylases. However, when looking up enzymes that have decarboxylase activity, it is not advisable to simply use EC4.1.1, because there are 11 other decarboxylases (Table 1) and 96 decarboxylating enzymes (Table 2). One of the enzymes that are not included in EC4.1.1, but which has decarboxylase activity, is EC1.1.1.42, which is named an "isocitrate dehydrogenase $(N A D P+)$ ", and has many synonyms including "oxalosuccinate decarboxylase", and a systematic name "isocitrate:NADP+oxidoreductase (decarboxylating)".

Another example that shows the importance of this analysis is EC3.2.1.183. An accepted name of this enzyme is "UDP- $N$-acetylglucosamine 2-epimerase (hydrolysing)", and the systematic name is " $U D P-N$-acetyl- $\alpha-D$-glucosamine hydrolase (2epimerising)". This enzyme is classified into EC3 hydrolases because of its hydrolysis activity, but the biological importance of this enzyme is its epimerisation activity. The main purpose of the Enzyme List is to give a unique name for a single catalytic entity. The unique name must end with the suffix -ase, and the suffix must not 
appear twice in a name. Therefore, when an enzyme has two activities, one of them is used with the suffix -ase, and the other is used with the suffix -ing.

To sum it up, the difference in the first digits of the EC numbers does not necessarily mean that the enzymes have no characteristics in common. In fact, however, many bioinformatics studies frequently rely on EC numbers, possibly resulting in erroneous interpretation in looking up the enzymatic function of interest, and in finding common characteristics from groups of enzymes or reactions. Here, we emphasize that the full value of the Enzyme List lies in the natural language descriptions, not in the IDs (i.e. EC numbers). We believe that this is a critical issue for a wide range of bioinformatics studies, including genome annotation and pathway analysis.

\subsection{Standardization and mapping of terminology}

The IUPAC recommendations of $1988^{6}$ indicate the nomenclature for organic chemical transformations and state that a transformation should be distinguished from a reaction; the full description of a reaction concerns all the reactants and all the products, whereas a transformation only concerns the changes in a particular molecule (Fig. 1). These recommendations use terminology that is different from that of the Enzyme List. For example, "dehydrogenation" (as dehydrogenase in the Enzyme List) is equivalent to "dihydroelimination" in the IUPAC recommendations of 1988. Taking another example from Fig. 1, "acylation" refers to a process in which a hydrogen atom of a substrate is replaced by an acyl group, irrespective of where the acyl group comes from or what the substrate is. This terminology is equivalent to the terminology "acyl-de-hydro-substitution" in IUPAC. These terms do not represent reactions but represent transformations.

On the other hand, the IUBMB's Enzyme List contains many concepts that are not included in the IUPAC recommendations of 1988. Some of the vocabulary does not represent transformations, but rather represent reactions (e.g. hydrolysis and phosphorolysis). Other terms have the structure "product + -forming", where the product is represented as the name of the compound (e.g. pyruvate-forming) or the functional group involved (e.g. aldehyde-forming). These terms do not represent transformations or reactions.

We collected the terms in the Enzyme List, and categorized them into those that represent reactions, transformations, and others. Consequently, standardized terms containing the suffixes -sis and -ation were mapped to reactions and transformations, respectively. Table 3 shows representative examples of this terminology mapping.

\subsection{Construction of the PIERO ontology}

The PIERO ontology was constructed by following the IUPAC recommendations of 1988, distinguishing between the descriptive terms for reactions and transformations. Among the objects already defined in KEGG, the REACTION and the RPAIR entries are equivalent to reactions and transformations, respectively. Some 
Table 3. Terminology mapping.

\begin{tabular}{ll}
\hline PIERO & \multicolumn{1}{c}{ Enzyme List } \\
\hline (a) Reactions & Hydrolase \\
Hydrolysis & Peptidase, protease, \\
Hydrolysis of peptide bond & Proteinase, etc. \\
& Glycosylase, glucosidase, etc. \\
Hydrolysis of glycosyl bond & Phosphatase \\
Hydrolysis of phosphate ester & ATPase \\
Hydrolysis of ATP & Glycosyltransferase \\
Phosphorolysis & Ligase \\
Phosphorolysis of glycosyl bond & Ligase, synthase, synthetase \\
Dehydration synthesis & Lyase \\
Synthesis & \\
Lysis & Oxidoreductase, reductase \\
(b) Transformations & Dehydrogenase \\
Oxidoreduction & Oxygenase, dioxygenase \\
Hydrogenation/dehydrogenation & Hydroxylase \\
Oxygenation/deoxygenation & Kinase, phosphatase, phospho-transferase, \\
Hydroxylation/dehydroxylation & phosphorylase \\
Phosphorylation/dephosphorylation & Pyrophosphorylase, diphosphatase \\
Pyrophosphorylation/depyrophosphorylation & Methyltransferase \\
Methylation/demethylation & Aminotransferase, deaminase, \\
Amination/deamination & Transaminase \\
Acylation/deacylation & Acyltransferase \\
Acetylation/deacetylation & Acetyltransferase \\
Hydration/dehydration & Hydratase, dehydratase \\
Cyclization/decyclization & Cyclase \\
Aldol reaction & Aldolase \\
Glycosylation/deglycosylation & Glycosyltransferase \\
Galactosylation/degalactosylation & Galactosyltransferase \\
Phosphorylation of hydroxy group & \\
Phosphorylation of carboxy group & Carboxylase, decarboxylase, \\
Carboxylation/decarboxylation & Isomerase, epimerase \\
Isomeration & Mutase \\
Intramolecular-migration & \\
(c) Product compound formation & Pyruvate-forming \\
Pyruvate formation & Phosphate-forming \\
Phosphate formation & \\
(d) Product substructure formation & Aldehyde-forming \\
Aldehyde-formation & to (a)thethl-forming \\
Carboxymethyl-formation & \\
\hline
\end{tabular}

Note: Vocabulary in the Enzyme List was mapped to (a) those representing overall reactions (reactions), (b) those representing partial reactions (transformations), (c) those representing the product compounds, and (d) those representing the product functional groups, and their suffixes were standardized into "-ation" or "-sis". 


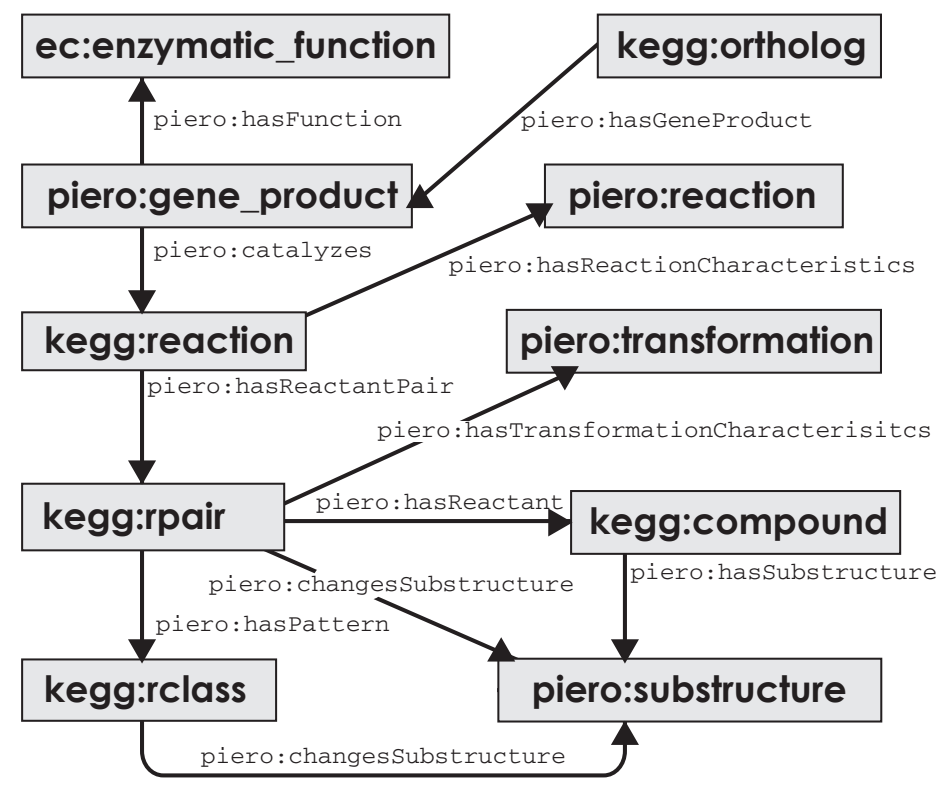

Fig. 2. Proposed ontological relationships in PIERO. The rectangles represent the classes, and the arrows represent the predicates that connect the subjects and the objects. "piero:", "ec:" and "kegg:" are the prefix of the classes and the predicates defined in PIERO, EC numbers, and KEGG.

additional concepts in $\mathrm{KEGG},{ }^{8} \mathrm{GO},{ }^{3}$ and $\mathrm{BiSSCat}^{11}$ were incorporated into PIERO, as follows (Fig. 2).

- A compound is a molecule whose structure can change during enzymatic reactions. Equivalent to the KEGG COMPOUND and other compound database entries.

- A substructure is a substructure or a functional group in a compound that has name(s) defined in IUPAC or IUBMB. Equivalent to the BiSSCat FGROUP entry.

- An rclass is a substructure that changes during the transformation, equivalent to the KEGG RCLASS entry.

- An enzymatic function is a subclass of a molecular function defined in GO, which is an experimentally characterized catalytic activity and the associated conditions mediated by the gene products. Equivalent to the EC number.

- A gene product is a substance (often a protein) with verified enzymatic function(s), and is encoded by a gene. A gene is equivalent to the KEGG GENES entry.

- An ortholog is a group of genes that share the same evolutionary origin and the same molecular function. Equivalent to the KEGG ORTHOLOGY entry.

The arrows between the classes represent the predicates, e.g. the arrow between gene product and reaction is "catalyzes", representing "A gene product catalyzes a reaction". In PIERO, the gene products have enzymatic functions, which are associated with EC numbers (and GO biological functions). We separated enzymatic 


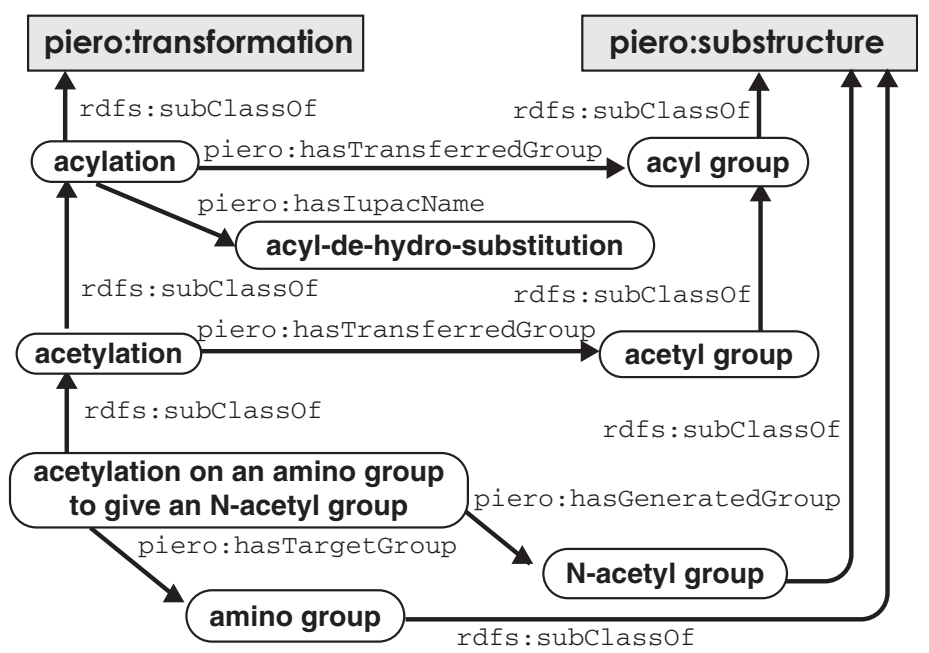

Fig. 3. Specialization of transformation terminology using substructures. The rectangles and arrows represent the classes and predicates, respectively. The rounded rectangles represent the specialized transformation terminology or substructures. "rdfs:" represent the prefix of the predicates defined in the RDF schema.

functions and reactions because some enzymes catalyze more than one reaction, and we attempted to deal with the common characteristics of those reactions, regardless of EC numbers.

A reaction consists of one or more transformations, and transformations are characterized by compounds, substructures, and transformation patterns (Fig. 2). The transformation terminology can be modified by using additional information (Fig. 3). For example, the transformation in Fig. 1(a) involves an acetylation, which is a specific term related to acylation. This relationship corresponds to that between the substructures acetyl group and acyl group. The use of the substructure or compound as a target group or a generated group gives a more specific description of the transformation. For example, the transformation in Fig. 1(a) can be regarded as a subclass of "acetylation on an amino group to yield an $\mathrm{N}$-acetyl group".

\subsection{Identifying putative enzymes from substrate-product relationships}

Here, we demonstrate some examples of applications of the PIERO ontology. The first situation mentioned in the Introduction section was formulated as a problem in identifying enzymes that putatively catalyze the transformation of L-lysine into N6-acetyl-L-lysine. These compounds are given the compound IDs C00047 and C02727 in KEGG, respectively.

When we simply want to identify the known reactions, exploring the path compound $->$ transformation $->$ reaction would be sufficient (Fig. 2). The transformation that contain both C00047 and C02727 is given the ID RP00263 in KEGG. The 
reactions that contain the transformation RP00263 are R00454, R00458, and R01620, which are equivalent to the reactions in Fig. 1(b).

When we required further reactions that include transformations such as "acylation of an amino group to yield an $N$-acyl group," exploring the path transformation $->$ substructure (Fig. 3) -> transformation pattern $->$ transformation -> reaction (Fig. 2) returned 299 reactions. When we specified more detailed information, such as "acetylation of an amino group to yield an $\mathrm{N}$-acyl group," 64 reactions were obtained.

\subsection{Extracting common characteristics from multiple genes}

The second situation in the Introduction was formulated as the problem of finding common transformations in given reactions, and it is as simple as exploring the path reaction -> transformation. When the given reactions were R00454, R00458, and R01620 (Fig. 1(b)), the obtained transformation was RP00263 (Fig. 1(a)).

As a further demonstration of the usefulness of PIERO, we analyzed the common characteristics of enzymatic reactions that are catalyzed by enzymes belonging to the same orthologous group. Table 4 shows some examples, where the orthologs were classified into those (a) sharing the same type of transformations, (b) catalyzing sequential reactions that share the same type of transformations, (c) catalyzing

Table 4. Common characteristics obtained from the orthologous enzymes mediating multiple functions.

\begin{tabular}{|c|c|c|c|}
\hline Common characteristics & Ortholog & EC number & Enzyme function \\
\hline \multicolumn{4}{|c|}{ (a) Sharing the transformations for the same type of bonds } \\
\hline \multirow[t]{6}{*}{ Phosphate esters } & K01103 & 2.7.1.105 & 6-phosphofructo-2-kinase \\
\hline & & 3.1 .3 .46 & Fructose-2,6-bisphosphate 2-phosphatase \\
\hline & K02203 & 2.7.1.39 & Homoserine kinase \\
\hline & & 3.1.3.3 & Phosphoserine phosphatase \\
\hline & K08073 & 2.7.1.78 & Polynucleotide $5^{\prime}$-hydroxyl-kinase \\
\hline & & 3.1.3.32 & Polynucleotide $3^{\prime}$-phosphatase \\
\hline \multirow[t]{2}{*}{ Glycosyl group } & K01196 & 2.4.1.25 & 4-alpha-glucanotransferase \\
\hline & & 3.2.1.33 & Amylo-alpha-1,6-glucosidase \\
\hline \multirow[t]{2}{*}{ Glycosyl group } & K05343 & 3.2 .1 .1 & Alpha-amylase \\
\hline & & 5.4 .99 .16 & Maltose glucosylmutase \\
\hline \multirow[t]{2}{*}{ Nucleotidyl group } & K12506 & 2.7 .7 .60 & MEP cytidylyltransferase \\
\hline & & 4.6.1.12 & MECDP-synthase \\
\hline \multirow[t]{2}{*}{ Amide bond } & K01460 & 3.5.1.78 & Glutathionylspermidine amidase \\
\hline & & 6.3 .1 .8 & Glutathionylspermidine synthase \\
\hline \multirow[t]{3}{*}{ Carbamoyl group } & K11540 & 2.1.3.2 & Aspartate transcarbamylase \\
\hline & & 3.5.2.3 & Dihydroorotase \\
\hline & & 6.3 .5 .5 & Carbamoyl-phosphate synthetase 2 \\
\hline \multirow[t]{3}{*}{ Deamination } & K00816 & 2.6.1.7 & Kynurenine - oxoglutarate transaminase \\
\hline & & 2.6.1.64 & Glutamine - phenylpyruvate transaminase \\
\hline & & 4.4.1.13 & Cysteine-S-conjugate beta-lyase \\
\hline \multirow[t]{4}{*}{ Thioester bond } & K00659 & 2.3.1.65 & Bile acid-CoA:amino acid N-acyltransferase \\
\hline & & 3.1 .2 .2 & Palmitoyl-CoA hydrolase \\
\hline & K05939 & 2.3.1.40 & Acyl-[ACP $]$ - phospholipid O-acyltransferase \\
\hline & & 6.2 .1 .20 & Acyl-acyl carrier protein synthetase \\
\hline
\end{tabular}


Table 4. (Continued)

\begin{tabular}{|c|c|c|c|}
\hline Common characteristics & Ortholog & EC number & Enzyme function \\
\hline Formyl group & K14652 & $\begin{array}{l}3.5 .4 .25 \\
4.1 .99 .12\end{array}$ & $\begin{array}{l}\text { GTP cyclohydrolase II } \\
\text { DHBP synthase }\end{array}$ \\
\hline Thiol/selenothiol group & K11717 & $\begin{array}{l}2.8 .1 .7 \\
4.4 .1 .16\end{array}$ & $\begin{array}{l}\text { Cysteine desulfurase } \\
\text { Selenocysteine lyase }\end{array}$ \\
\hline Hydrogen sulfide & K13034 & $\begin{array}{l}2.5 .1 .47 \\
4.4 .1 .9\end{array}$ & $\begin{array}{l}\text { Cysteine synthase } \\
\text { L-3-cyanoalanine synthase }\end{array}$ \\
\hline Aldol reaction & K16305 & $\begin{array}{l}2.2 .1 .11 \\
4.1 .2 .13\end{array}$ & $\begin{array}{l}\text { 6-deoxy-5-ketofructose } 1 \text {-phosphate synthase } \\
\text { Fructose-bisphosphate aldolase }\end{array}$ \\
\hline Triphosphate of ATP & K01947 & $\begin{array}{l}2.7 .1 .33 \\
6.3 .4 .15\end{array}$ & $\begin{array}{l}\text { Type III pantothenate kinase } \\
\text { Biotin-[acetyl-CoA-carboxylase] ligase }\end{array}$ \\
\hline \multicolumn{4}{|c|}{ (b) Catalyzing sequential reactions that share the same type of transformations } \\
\hline Hydrogenation of porphyrins & K02302 & $\begin{array}{l}1.3 .1 .76 \\
2.1 .1 .107 \\
4.99 .1 .4\end{array}$ & $\begin{array}{l}\text { Precorrin-2 dehydrogenase } \\
\text { Uroporphyrin-III C-methyltransferase } \\
\text { Sirohydrochlorin ferrochelatase }\end{array}$ \\
\hline Oxygenation & K17863 & $\begin{array}{l}1.13 .11 .60 \\
5.4 .4 .5\end{array}$ & $\begin{array}{l}\text { Linoleate 8R-lipoxygenase } \\
\text { Fructose-bisphosphate aldolase }\end{array}$ \\
\hline Cyclization of terpenes & K12928 & $\begin{array}{l}4.2 .3 .42 \\
5.5 .1 .14\end{array}$ & $\begin{array}{l}\text { Aphidicolan-16beta-ol synthase } \\
\text { Syn-copalyl-diphosphate synthase }\end{array}$ \\
\hline \multicolumn{4}{|c|}{ (c) Catalyzing sequential reactions that do not share the same type of transformations } \\
\hline Fatty acid metabolism & K01825 & $\begin{array}{l}1.1 .1 .35 \\
4.2 .1 .17 \\
5.1 .2 .3 \\
5.3 .3 .8\end{array}$ & $\begin{array}{l}\text { 3-hydroxyacyl-CoA dehydrogenase } \\
\text { Enoyl-CoA hydratase } \\
\text { 3-hydroxybutyryl-CoA epimerase } \\
\text { Enoyl-CoA isomerase }\end{array}$ \\
\hline Starch metabolism & K16055 & $\begin{array}{l}2.4 .1 .15 \\
3.1 .3 .12\end{array}$ & $\begin{array}{l}\text { Trehalosephosphate-UDP glucosyltransferase } \\
\text { Trehalose-phosphatase }\end{array}$ \\
\hline \multicolumn{4}{|c|}{ (d) Acting on similar substrates without sharing the same type of transformations } \\
\hline DNA/RNA modification & K15719 & $\begin{array}{l}3.6 .4 .12 \\
5.99 .1 .3\end{array}$ & $\begin{array}{l}\text { DNA helicase } \\
\text { DNA topoisomerase }\end{array}$ \\
\hline Protein modification & K03170 & $\begin{array}{l}2.3 .1 .48 \\
3.2 .1 .169\end{array}$ & $\begin{array}{l}\text { Histone acetyltransferase } \\
\text { Protein O-GlcNAcase }\end{array}$ \\
\hline Shikimate pathway & K13829 & $\begin{array}{l}2.7 .1 .71 \\
4.2 .3 .4\end{array}$ & $\begin{array}{l}\text { Shikimate kinase } \\
\text { 3-dehydroquinate synthase }\end{array}$ \\
\hline
\end{tabular}

sequential reactions that do not share the same type of transformations, and (d) acting on similar substrates without sharing the same type of transformations. Here, the same type of transformation means those transformations that generate or cleave the same type of bonds. Among the 102 orthologs that mediate the reactions with different EC numbers at the first digit, 38 and eight orthologs were classified as (c) and (d), respectively, and no common characteristics was found in five of the orthologs. The remaining 51 orthologs were found to have some common characteristics related to the type of transformations.

In this paper, we only showed representative examples, i.e. the common characteristics of reactions that have different EC classes (the first digits of EC numbers), but which are mediated by the same orthologs. However, this analysis could be applied to many other groups of reactions, e.g. the reactions that have different EC 
numbers (or subclasses), but which are mediated by the same orthologs or by the same protein domains, etc.

\section{Conclusion}

In this study, we introduced the concept of transformation based on the IUPAC recommendation regarding the characterization of the Enzyme List of IUBMB, which facilitates identification of possible reactions from a transformation, and for finding common characteristics from a given set of reactions. Although we used the KEGG database to design the proposed PIERO ontology, it has links to genes, orthologs, EC numbers, and compounds in other databases. We intend to further develop the PIERO ontology, effectively using the IUBMB's Enzyme List that is carefully managed in the form of natural language, and which should allow elucidation of the relationship between genomics and metabolomics from the biochemical changes of interest to users.

The PIERO ontology has been implemented as an OWL ontology and is available via a GenomeNet SPARQL endpoint (http://reactionontology.org/), where the users can make queries using the SPARQL language. ${ }^{13}$ Recent years, RDF is gathering more attention in the bioinformatics community to describe various types of data, and some RDF-related tools (including SPARQL endpoints) are becoming available. ${ }^{14-16}$ To date, these are still developing technologies, and many studies attempt to improve the calculation efficiency and the usability. ${ }^{17-19}$ Our SPARQL endpoint also leaves room to improve, although the continuing development of the ontology data and the related technologies would enable more complex queries with better usability in the foreseeable future.

\section{Acknowledgments}

We thank all the organizers and participants of the BioHackathon series ${ }^{19}$ for inspiring exchanges of ideas and for technical support. Computational resources were provided by the Bioinformatics Center, Institute for Chemical Research and the Super Computer Laboratory, Kyoto University. This study was funded by the Ministry of Education, Culture, Sports, Science and Technology of Japan, the Japan Science and Technology Agency; and the Japan Society for the Promotion of Science, MEXT Kakenhi (25108714).

\section{References}

1. Mittler R, Shulaev V, Functional genomics, challenges and perspectives for the future, Physiologia Plantarum 148:317-321, 2013.

2. McDonald AG, Tipton KF, Fifty-five years of enzyme classification: Advances and difficulties, FEBS J 281:583-592, 2013.

3. Ashburner M, Ball CA, Blake JA, Botstein D, Butler H, Cherry JM, Davis AP, Dolinski K, Dwight SS, Eppig JT, Harris MA, Hill DP, Issel-Tarver L, Kasarskis A, Lewis S, 
Matese JC, Richardson JE, Ringwald M, Rubin GM, Sherlock G, Gene ontology: Tool for the unification of biology. The Gene Ontology Consortium, Nat Genet 25:25-29, 2000.

4. Nakabayashi R, Saito K, Metabolomics for unknown plant metabolites, Anal Bioanal Chem 405:5005-5011, 2013.

5. Noy NF, Shah NH, Whetzel PL, Dai B, Dorf M, Griffith N, Jonquet C, Rubin DL, Storey MA, Chute CG, Musen MA, BioPortal: Ontologies and integrated data resources at the click of a mouse, Nucleic Acids Res 37:W170-W173, 2009.

6. Jones RAY, Bunnett JF, Nomenclature for organic chemical transformations, Pure Appl Chem 61:725-768, 1989.

7. Hastings J, de Matos P, Dekker A, Ennis M, Harsha B, Kale N, Muthukrishnan V, Owen G, Turner S, Williams M, Steinbeck C, The ChEBI reference database and ontology for biologically relevant chemistry: Enhancements for 2013, Nucleic Acids Res 41:D456D463, 2013.

8. Kanehisa M, Goto S, Sato Y, Kawashima M, Furumichi M, Tanabe M, Data, information, knowledge and principle: Back to metabolism in KEGG, Nucleic Acids Res 42: D199-D205, 2014.

9. Kotera M, Goto S, Kanehisa M, Predictive genomic and metabolomic analysis for the standardization of enzyme data, Perspect Sci 1:24-32, 2014.

10. Muto A, Kotera M, Tokimatsu T, Nakagawa Z, Goto S, Kanehisa M, Modular architecture of metabolic pathways revealed by conserved sequences of reactions, J Chem Inf Model 53:613-622, 2013.

11. Kotera M, McDonald A, Boyce S, Tipton K, Functional group and substructure searching as a tool in metabolomics, PLoS ONE 3:e1537, 2008.

12. Kotera M, Tabei Y, Yamanishi Y, Moriya Y, Tokimatsu T, Kanehisa M, Goto S, KCF-S: KEGG chemical function and substructure for improved interpretability and prediction in chemical bioinformatics, BMC Syst Biol 7(Suppl 6):S2, 2013.

13. Quilitz B, Leser U, Querying Distributed RDF Data Sources with SPARQL, Lecture Notes in Computer Science, The Semantic Web: Research and Applications 5021:524$538,2008$.

14. Deus HF, Veiga DF, Freire PR, Weinstein JN, Mills GB, Almeida JS, Exposing the cancer genome atlas as a SPARQL endpoint, J Biomed Inform 43:998-1008, 2010.

15. Anguita A, Garcia-Remesal M, de la Iglesia D, Maojo V, NCBI2RDF: Enabling full RDFbased access to NCBI databases, Biomed Res Int 2013:983805, 2013.

16. Faulconbridge A, Burdett T, Brandizi M, Gostev M, Pereira R, Vasant D, Sarkans U, Brazma A, Parkinson H, Updates to BioSamples database at European Bioinformatics Institute, Nucleic Acids Res 42:D50-D52, 2014.

17. Wu H, Fujiwara T, Yamamoto Y, Bolleman J, Yamaguchi A, BioBenchmark Toyama 2012: An evaluation of the performance of triple stores on biological data, J Biomed Semantics 5:32, 2014.

18. Kawano S, Watanabe T, Mizuguchi S, Araki N, Katayama T, Yamaguchi A, TogoTable: Cross-database annotation system using the Resource Description Framework (RDF) data model, Nucleic Acids Res 42:W442-W448, 2014.

19. Katayama T, Wilkinson MD, Aoki-Kinoshita KF, Kawashima S, Yamamoto Y, Yamaguchi A, Okamoto S, Kawano S, Kim J, Wang Y, Wu H, Kano Y, Ono H, Bono H, Kocbek S, Aerts J, Akune Y, Antezana E, Arakawa K, Aranda B, Baran J, Bolleman J, Bonnal RJP, Buttigieg PL, Campbell MP, Chen Y, Chiba H, Cock PJA, Cohen KB, Constantin A, Duck G, Dumontier M, Fujisawa T, Fujiwara T, Goto N, Hoehndorf R, Igarashi $\mathrm{Y}$, Itaya $\mathrm{H}$, Ito $\mathrm{M}$, Iwasaki $\mathrm{W}$, Kalas $\mathrm{M}$, Katoda $\mathrm{T}$, Kim T, Kokubu A, Komiyama Y, Kotera M, Laibe C, Lapp H, Lutteke T, Marshall MS, Mori T, Mori H, Morita M, Murakami K, Nakao M, Narimatsu H, Nishide H, Nishimura Y, 
Nystrom-Persson J, Ogishima S, Okamura Y, Okuda S, Oshita K, Packer NH, Prins P, Ranzinger R, Rocca-Serra P, Sansone S, Sawaki H, Shin S, Splendiani A, Strozzi F, Tadaka S, Toukach P, Uchiyama I, Umezaki M, Vos R, Whetzel PL, Yamada I, Yamasaki C, Yamashita R, York WS, Zmasek CM, Kawamoto S, Takagi T, BioHackathon series in 2011 and 2012: penetration of ontology and Linked Data in life science domains, J Biomed Semantics 5:5, 2014.

Masaaki Kotera is an Associate Professor at Tokyo Institute of Technology, Japan. He received PhD Science in Bioinformatics in 2005 from Kyoto University, Japan, worked as Research Fellow in Trinity College Dublin, Ireland, during 20052008, and as Assistant Professor of Kyoto University during 2008-2014. His principal research interests lie in the field of bioinformatics and cheminformatics, with a concentration on enzyme proteins and reactions.

Yosuke Nishimura is a PhD student at Kyoto University, Japan. He studies wide varieties of research fields including ontology, cheminformatics, and bioinformatics.

Zen-Ichi Nakagawa is a project member of KEGG (Kyoto Encyclopedia of Genes and Genomes) database. He works on curating enzyme reaction and chemical compound data in KEGG.

Ai Muto is an Assistant Professor at Nara Institute of Science and Technology, Japan. She received PhD Science in Bioinformatics from Kyoto University in 2013. Her research topics include enzyme reaction similarity from chemical point of view.

Yuki Moriya is a Project Researcher in Database Center for Life Science (DBCLS), Japan. He works on many bioinformatics webservers for genome annotation and metabolic pathway prediction.

Shinobu Okamoto is an Associate Professor in DBCLS, Japan. He works on the standardization of biological metadata and the design of applications using Semantic Web technologies.

Shuichi Kawashima is an Assistant Professor in DBCLS, Japan. He works on the integration of life science databases and software applications using Semantic Web and bioinformatics technologies. 
Toshiaki Katayama is an Assistant Professor in DBCLS, Japan. He works on the integration of genomic information using Semantic Web technologies, and the software development.

Toshiaki Tokimatsu is a Project Researcher in DBCLS, Japan. He received PhD for Wood Biochemistry in Agriculture from Kyoto University in 1999. He works on plant genomes and metabolome information involving the synthesis of secondary metabolites.

Minoru Kanehisa is a Professor of Kyoto University. He received PhD in Physics from the University of Tokyo in 1976. He established the KEGG database for understanding the biological system from molecular-level information generated by genome sequencing and other high-throughput experimental technologies.

Susumu Goto is an Associate Professor of Kyoto University. He received PhD from Kyushu University in 1994. He established many bioinformatics databases and webservers in GenomeNet, a network of database and computational services for genome research and related research areas in biomedical sciences. 